\title{
Mixed-modality psychophysical scaling: Sequential dependencies and other properties
}

\author{
LAWRENCE M. WARD \\ University of British Columbia, Vancouver, B. C. V6T 1W5, Canada
}

\begin{abstract}
J. C. Stevens and Marks's (1980) method of magnitude matching and a derivative, category matching, are examined in two experiments. Category matching produces highly similar matching functions to those of magnitude matching. The two methods are both subject to significant sequential dependencies, which, however, should not affect exponents of matching functions. Such mixed-modality scaling methods are also useful for theory testing. In the present case, responses were assimilated to the immediately previous response (different modality stimulus) but contrasted with the stimulus (same modality) two trials back in the sequence. The dependency of the response-response dependency on $S_{n}-S_{n-k}$ was independent of stimulus modality. However, the usual dependency of the coefficient of variation of ratios of successive responses on $S_{n}-S_{n-k}$ was not found. These results support the class of theories in which assimilative response-response dependencies and contrastive response-stimulus dependencies arise from different mechanisms, and disconfirm those in which both effects arise in a linked fashion from a single mechanism.
\end{abstract}

J. C. Stevens and Marks (1980) introduced a new method of producing cross-modality matching functions. They simply asked subjects to do ordinary magnitude estimates (on a common scale) of stimuli from the modalities to be matched, presented alternately (e.g., light-sound-light-sound), and then used a mathematical procedure to generate cross-modality matching functions from the resulting magnitude estimates. This method, called magnitude matching, was argued to have several advantages over the traditional cross-modality matching procedure while generating similar matching functions.

Magnitude matching is one member of a larger set that I will call mixed-modality psychophysical scaling. Clearly, cross-modality matching functions can be generated from any kind of accepted psychophysical scaling method performed alternately on stimuli from two or more sensory continua. For example, a method called category matching can be created by having subjects make category judgments (on a common scale) of stimuli from two or more modalities. In a similar way, matching functions could be generated from magnitude or category productions, ratio or difference estimates, or even crossmodality matches made on a third, common, response continuum. Although not all of these possible

\footnotetext{
This research was supported by Grant A9958 from the Natural Sciences and Engineering Research Council of Canada. I thank Barry Chew, Titus Chou, and Paul Pellicano for help with running the subjects, Klaus and Marsha Schroeder for help with data analyses, and Larry Marks for helpful comments pertaining particularly to the Appendix. Send reprint requests to: Lawrence M. Ward, Department of Psychology, University of British Columbia, 2075 Wesbrook Place, Vancouver, B. C. V6T 1W5, Canada.
}

methods will share the advantages of magnitude matching, some may prove superior. For example, category matching seems to emphasize the necessity for responding on a common scale more than does magnitude matching, since responses are limited to particular category labels.

Whether superior or inferior in production of useful cross-modality matching functions, mixed-modality methods may prove useful in testing theories about psychophysical judgment processes. For example, several recent theories of psychophysical judgment attempt to explain the sequential dependencies that are ubiquitous in psychophysical scaling methods. In magnitude estimations and productions, category judgments, and cross-modality matches, the current response is typically assimilated to previous responses and contrasted with previous stimuli (Green, Luce, \& Duncan, 1977; Ward, 1972, 1973, 1975). Mixedmodality techniques may provide an empirical context in which to distinguish competing theories of the origin of such effects.

One theory that has generated many new results is the response ratio hypothesis of Luce and Green (1974). They proposed that magnitude estimation responses are made according to

$$
\frac{R_{n}}{R_{n-1}}=C \frac{X\left(S_{n}\right)}{X^{*}\left(S_{n-1}\right)}
$$

where $R_{n}$ and $R_{n-1}$ are random variables representing a numerical response on Trials $n$ and $n-1, C$ is a constant, $X\left(S_{n}\right)$ and $X^{*}\left(S_{n-1}\right)$ are random variables representing the internal representations of the stimuli on Trials $n$ and $n-1$, and $S_{n}$ and $S_{n-1}$ are 
the nominal values of the stimuli on Trials $\mathrm{n}$ and $\mathrm{n}-1$. Assuming that

$$
X\left(S_{n}\right)=X^{*}\left(S_{n}\right)=k I_{n}^{m},
$$

where $I_{n}$ is a random variable representing the physical intensity of the stimulus on Trial $n$, this model results in

$$
\begin{aligned}
\log R_{n} \\
=m \log I_{n}-b \log I_{n-1}+d \log R_{n-1}+\log C^{\prime} .
\end{aligned}
$$

As can be seen in Equation 3, both assimilation of $R_{n}$ to $R_{n-1}$ (d is positive) and contrast of $R_{n}$ with $I_{n-1}$ (b is negative) are predicted from this simple model. It is also clear that both effects arise from the same mechanism. Although this simple version of the response ratio hypothesis has been disconfirmed (Jesteadt, Luce, \& Green, 1977), more elaborate models based on its use under special conditions have been proposed to explain the details of magnitude estimation data (e.g., Luce, Baird, Green, \& Smith, 1980). All such models share the property that assimilation and contrast effects are generated by a single mechanism so that they are always associated.

A second class of models has the two types of sequential dependency generated by different mechanisms. An example is the fuzzy judgment model of Ward (1979). In this model, magnitude estimations, category judgments, and cross-modality matches are all characterized by an attempt by the subject to first categorize the stimulus. To do this, a cross-correlation is calculated between the internal representation of the stimulus (a fuzzy set over the possible sensation levels-Zadeh, 1965) and a set of category prototypes (also fuzzy sets over sensation levels). Usually more than one category achieves an acceptable crosscorrelation, creating an "indifference set." Choice of a single category from the indifference set in a nonrandom (biased) way (to be nearest the last category used) is hypothesized to cause the assimilation of $R_{n}$ to $R_{n-1}$. Contrast of $R_{n}$ with $S_{n-1}$ is hypothesized to occur for a different reason. Because of the excitatory-center/inhibitory-surround nature of the fuzzy internal representations of the stimuli, overlapping representations (e.g., of $S_{n}$ and $S_{n-1}$ ) "repel" each other (contrast effect) (see, e.g., Levine \& Grossberg, 1976). A mathematization of this model, in which the contrast effect appears during the formation of the internal representation of $S_{n}$, the assimilation effect appears during the choice of category label, and both are passed on through appropriate matching stages to determine $R_{n}$, results in an equation much like Equation 3 (Ward, 1979). However, the coefficients of $R_{n-1}, I_{n}$, and $I_{n-1}$ are independently determined in the equation arising from the fuzzy judgment model, while the response ratio hy- pothesis predicts values of $b=-m$ and $d=1$ for Equation 3, reflecting the derivation from Equations 1 and 2.

Mixed-modality psychophysical scaling can provide data relevant to empirically distinguishing between these two general classes of models. To see this, consider a sequence of magnitude estimations of lights alternated with sounds, viz:

$$
L_{n-2} R_{n-2} S_{n-1} R_{n-1} L_{n} R_{n} \text {, }
$$

where $L_{n-k}$ represents a light stimulus, $S_{n-k}$ a sound stimulus, and $R_{n-k}$ a response, on Trial $n-k$. Consider generation of $R_{n}$ in terms of the response ratio hypothesis. If internal representations of lights and sounds are commensurate, then application of Equation 1 to the $S_{n-1} R_{n-1} L_{n} R_{n}$ sequence produces assimilation of $R_{n}$ to $R_{n-1}$ and contrast with $S_{n-1}$. If not, then application of Equation 1 must be to the sequence $L_{n-2} R_{n-2} \ldots L_{n} R_{n}$, ignoring the intervening sound stimulus and the response to it. This produces assimilation to $R_{n-2}$ and contrast with $L_{n-2}$. Application to such sequences as $L_{n-2} \ldots$ $R_{n-1} L_{n} R_{n}$ makes no sense, since, for Equation 1 to produce a reasonable $R_{n}, R_{n-k}$ must be the response to the stimulus that enters into $X\left(S_{n}\right) / X^{*}\left(S_{n-k}\right)$, not a response to some other, unrelated, stimulus. Thus, this model and any based on it predict both assimilation and contrast only to associated stimuli and responses.

Now, consider "dual mechanism" theories like the fuzzy judgment model. Here, the category choice mechanism would produce assimilation of $R_{n}$ to $\mathbf{R}_{\mathbf{n}-1}$ (attempt to make current category label as similar as possible to previous category label). However, the stimulus contrast effect would most likely be with $L_{n-2}$, since it is of the same modality as $L_{n}$. It would be surprising if $R_{n}$ was contrasted with $S_{n-1}$, since, presumably, internal representations of light and sounds would occur in different brain regions (primary or secondary sensory projection areas?) and thus should not overlap and should not produce contrast. Most such imaginable stimulus contrast mechanisms would be similarly modality specific. So, if a pattern of assimilation to responses separately from contrast with the stimuli that gave rise to those responses were observed in magnitude matching data, a "dual mechanism" theory of judgment would be supported. If the effects occurred only with linked stimuli and responses, the single-mechanism theories would be more parsimonious, and perhaps preferable.

The present experiments had several purposes. The first was to investigate in detail the properties of magnitude matching data, especially sequential dependencies, since the method has been argued to be superior to other methods of psychophysical scaling, particularly in its freedom from bias (J. C. Stevens \& Marks, 1980). Second, since the method of cate- 
gory matching seems to be a natural extension of magnitude matching, perhaps with some advantages, I wanted to investigate the method and compare it with magnitude matching. Third, I wanted to use the mixed-modality property of both methods to attempt to distinguish between the two classes of models, outlined above, that attempt to account for sequential dependencies in psychophysical judgments.

\section{METHOD}

\section{Experiment 1: Magnitude Matching}

Subjects. Eight subjects with normal or corrected-to-normal vision and no known hearing defects participated for pay. Six were females and two were males.

Apparatus. A sequence of alternating sounds and lights was presented for judgment, as in the procedure of J. C. Stevens and Marks (1980). For the light stimuli, light from a 500-W bulb in a Kodak Carousel projector was led through infrared absorbing glass and a 1.0-log-unit neutral density filter, then through a set of logic-selectable neutral density filters, a fiber-optic light guide, two panes of IAC chamber glass, and a sheet of tracing paper, to form a $1.5-\mathrm{deg}$ dot of approximately uniform luminance. The dot appeared at about eye level on the surface of the chamber wall facing the subject. Luminances were measured using an SEI visual photometer that had been calibrated against a Model 1980A-PL Pritchard photometer. The 10 light intensities used were: $167.6,84.00,42.10,21.10,10.58,5.300,2.656,1.331,0.667$, $0.334 \mathrm{~cd} / \mathrm{m}^{2}$. Adjacent stimuli were thus $.3 \log$ units $(3 \mathrm{~dB})$ apart. For the sound stimuli, a 1,000-Hz sine-wave signal from a General Radio signal generator was led through one of a bank of logicselectable potentiometers, then amplified and gated by an electronic switch with rise and fall times set to approximately $50 \mathrm{msec}$. The resulting amplitudes were delivered diotically through stereo earphones (Sharpe Pro HA 660). The sound intensities were measured at the earphones with a precision sound meter (General Radio). Total measured sound pressure levels (right ear plus left ear) used were: $11.25,6.324,3.556,2.000,1.125,0.632,0.356$, $0.200,0.113$, and $0.063 \mathrm{dyne} / \mathrm{cm}^{2}$. Adjacent stimuli were thus separated by $.25 \log$ unit $(5 \mathrm{~dB})$. The ranges of light and sound intensities were selected so as to be roughly equal and convenient to generate. They are in the ranges used by J. C. Stevens and Marks (1980).

A PDP-11/10 computer system controlled the presentation of the stimuli and recorded the responses, which were indicated by subjects on a standard computer keyboard (illuminated by a dim red light). Stimuli were presented for 1 sec. Subjects sat in a dark, Model 250 IAC chamber while making their judgments.

Procedure. The subjects served in five approximately 1-h sessions. In the first, they received the instructions, were shown how to use the keyboard, made a few practice magnitude estimations of line lengths ranging from about 1 to $100 \mathrm{~cm}$, and made 200 practice magnitude estimations of the light and sound stimuli (100 of each type) presented alternately at randomly selected intensities. In each of the four subsequent sessions, the subjects made 300 magnitude estimations of the light and sound stimuli (150 of each type) for a total of 1,200 judgments, 600 each of light and sound (about 60 per intensity level).

Before each series of judgments, the subjects sat for $2 \mathrm{~min}$ in the dark (except for keyboard light) IAC chamber. The interval between presentations of light stimuli was $10-15 \mathrm{sec}$, depending on the subject's response latency on the intervening responses. This interval probably did not allow full dark adaptation after presentation of the most intense lights, but, as will be seen, this would not affect the general conclusions. Better control of this factor should, of course, be attained in diagnostic situations (cf. J. C. Stevens \& Marks, 1980).

The following instructions, adapted from those of J. C. Stevens and Marks (1980), were presented to subjects: "In this experiment you will be asked to judge several brightnesses of a light and several loudnesses of a sound. You do this by assigning to each event a number that best seems to match its intensity. To indicate the general range of numbers, a tone $\left(0.632 \mathrm{dyne} / \mathrm{cm}^{2}\right)$ will be presented first to whose loudness is assigned the number 100 (you do not have to respond to this first tone) as if you had called it 100 yourself on the first trial. You may use any integer from 1 to 32767 (32767 is the largest integer the computer could handle). Do not use zero and do not use decimals. Try to judge brightness and loudness on a common scale of intensity. Thus if a given light and a given sound seem to have the same intensity they should be given the same number. (This may never happen but conceivably it could). Light will always be alternated with sound (after the first sound) but the particular intensities of each will vary randomly. It is very important to assume that the absolute sizes of the numbers and the range of the numbers needed to match the loudnesses may be quite different from those needed to match the brightnesses. In other words try to make an 'absolute' match of the number and the sensation intensity in each presentation."

\section{Experiment 2: Category Matching}

Subjects. Eight different subjects with normal or corrected-tonormal vision and no known hearing defects participated for pay. Four were females and four were males.

Apparatus. All stimuli and equipment were identical to those used in Experiment 1.

Procedure. The procedure was identical to that of Experiment 1 except that subjects made category judgments instead of magnitude estimations of the stimuli. Thus, there was no magnitudeestimation-of-line-lengths practice in the first session, and subjects were presented with the four extreme stimuli instead of the 0.632 dyne $/ \mathrm{cm}^{2}$ sound at the beginning of each series of judgments. Also, subjects made only 100 practice judgments in the first session in this experiment.

The following instructions were given to subjects: "In this experiment you will be asked to judge several brightnesses of a light and several loudnesses of a sound. You will do this by making category judgments using a common set of categories. There will be 10 categories of sensory intensity into which your judgments could be placed, so you will assign to each light and sound a number from 1 to 10 (use 0 for 10) inclusive that describes the category into which it falls. To give you an idea of the extremes of the stimuli the two sounds (highest and lowest) and the two lights (highest and lowest) will be presented in the beginning of the experiment. Call the most intense appearing of these four a 10 and the least intense appearing a 1 . IE, try to divide the interval between these extreme intensities into ten equal intervals, with the most intense in category ten $(0)$ and the least intense in category one (1). Note that you do not have to respond to these first four stimuli but you may do so if you wish. Following these stimuli will be those that you will be judging. Either the light or the sound, but not both, will come on at one time. After you have made your judgment of its intensity by pressing a number from 1 to 0 on the keyboard, you must press the "]" terminal key and another stimulus will be presented to you. The stimuli will alternate between light and sound of a certain intensity. Note that the intensities will come at random, IE, a bright light will not necessarily be followed by the loudest sound."

\section{RESULTS AND DISCUSSION}

\section{Stevens-Marks Analyses}

Despite several differences between the procedures, the data of Experiment 1 resemble closely those of J. C. Stevens and Marks (1980). The top panel of Figure 1 shows the cross-modality matching function relating light and sound generated from the data of 


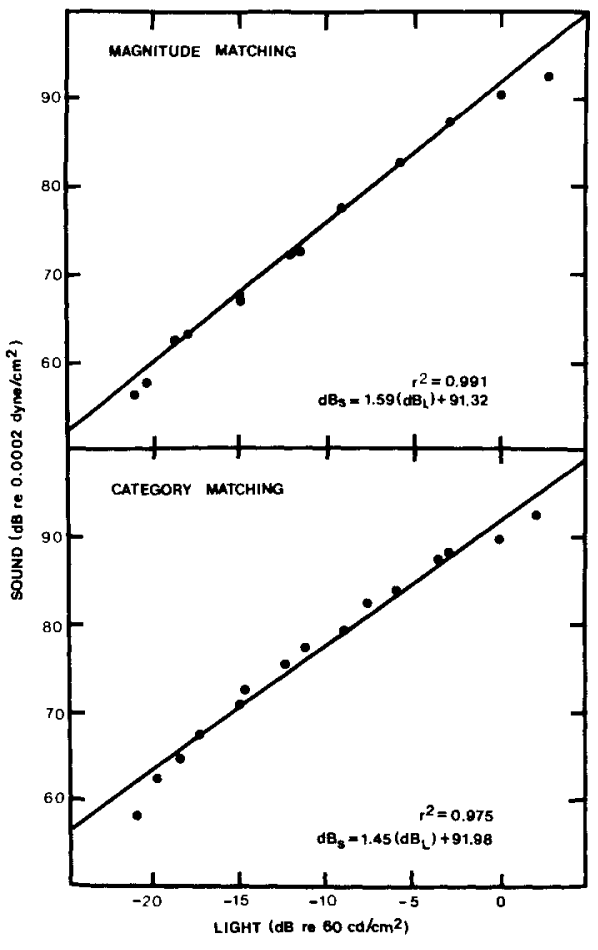

Figure 1. Average $(n=8)$ cross-modality matching functions generated from magnitude (top) and category (bottom) matching data.

Experiment 1 according to the procedure of J. C. Stevens and Marks (1980). This figure represents the average function over all of the subjects, but individual functions were highly similar. All functions display some curvature, as did those of J. C. Stevens and Marks (1980), but are reasonably well fitted by simple power functions (straight lines in decibel coordinates). The slopes of the matching functions ${ }^{1}$ of individual subjects averaged 1.60 (1.60 for the averaged data). This is somewhat larger than the $1.0 \mathrm{ex}-$ pected from the standard matching equation rendered in decibels and using typical (S. S. Stevens, 1975) power function exponents of .33 for light intensity and .67 for sound pressure. However, it is exactly the value predicted from that analysis when estimates of the separate power function exponents obtained for light and sound (average $\gamma$ in Table 1) are substituted for the more typical exponents (see Appendix). The average exponent for sound (.518) is smaller than usual, and that for light (.415) is larger than usual. The larger light exponent could have been caused by a less than complete state of dark adaptation (which would have decreased the apparent stimulus range) throughout the experiment. The sound exponent obtained is in the range of typical values reported (cf. Marks, 1974). Another possibility (suggested by a reviewer) is that subjects had a tendency to equate the ranges of numbers given to both sets of stimuli. Further research along the lines of that of J. C. Stevens and Marks (1980), in which one range is held constant and the other varied, might clarify this matter.

The average and individual data of Experiment 2 are very similar to those of Experiment 1 . The bottom panel of Figure 1 shows the average category matching function. Individual functions were highly similar. The average function displays more curvature than that for magnitude matching but is still well described by a power function. Moreover, the average slope of the individual category matching functions (1.47) is not significantly different from that of individual magnitude matching functions $[\mathrm{t}(14)=.739]$. Thus, although psychophysical power functions from magnitude estimation and category judgment typically do not have the same exponents (Marks, 1974; Ward, 1971), magnitude matching and category matching functions do. Not only does this approach diminish some departures of the psychophysical functions from the power law (J. C. Stevens \& Marks, 1980), but also it demonstrates that both category judgment and magnitude estimation can generate reliable, valid, and equivalent information.

\section{Sequential Dependencies}

Multiple regression analyses. A useful summary of sequential dependencies is provided by estimation of $\gamma, \alpha_{\mathrm{i}}$, and $\beta_{\mathrm{k}}$ in

$$
\begin{aligned}
\log R_{n}= & \gamma \log I_{n}+\sum_{i=1}^{M} \alpha_{i} \log I_{n-i}+\sum_{k=1}^{N} \beta_{k} \log R_{n-k} \\
& +\delta+\varepsilon
\end{aligned}
$$

(Jesteadt, Luce, \& Green, 1977; Ward, 1979). Here $M=10$ and $N=10$. Table 1 summarizes the results of this analysis of the data of the magnitude matching experiment. Only results for $\mathbf{M}=2$ and $\mathrm{N}=2$ are reported, since higher values seldom yielded significant effects for individual subjects. ${ }^{2}$ In interpreting Table 1, remember that light and sound alternate in the sequence of trials. Also notice that responses $\left(R_{n}\right)$ to light and sound stimuli are analyzed separately. Thus, for the responses to sound stimuli, $I_{n}$ is a sound stimulus, $R_{n-1}$ is a response to a light stimulus, $I_{n-1}$, $R_{n-2}$ is a response to a sound stimulus, $I_{n-2}$, and vice versa for responses to light stimuli. Thus, the results of Table 1 indicate that, as usual, $I_{n}$ has a significant effect on $R_{n}$ for both light and sound responses, as do $R_{n-1}, R_{n-2}$, and $I_{n-2}$. However, $I_{n-1}$, the stimulus on the preceding trial, which is of a different modality from that of the current stimulus, exerts no effect on the response to the current stimulus. Thus, although the current response is assimilated toward previous responses (positive sign of $\beta_{1}$ and $\beta_{2}$ ) regard- 
Table 1

Results of Multiple-Regression Analyses of Magnitude Matching

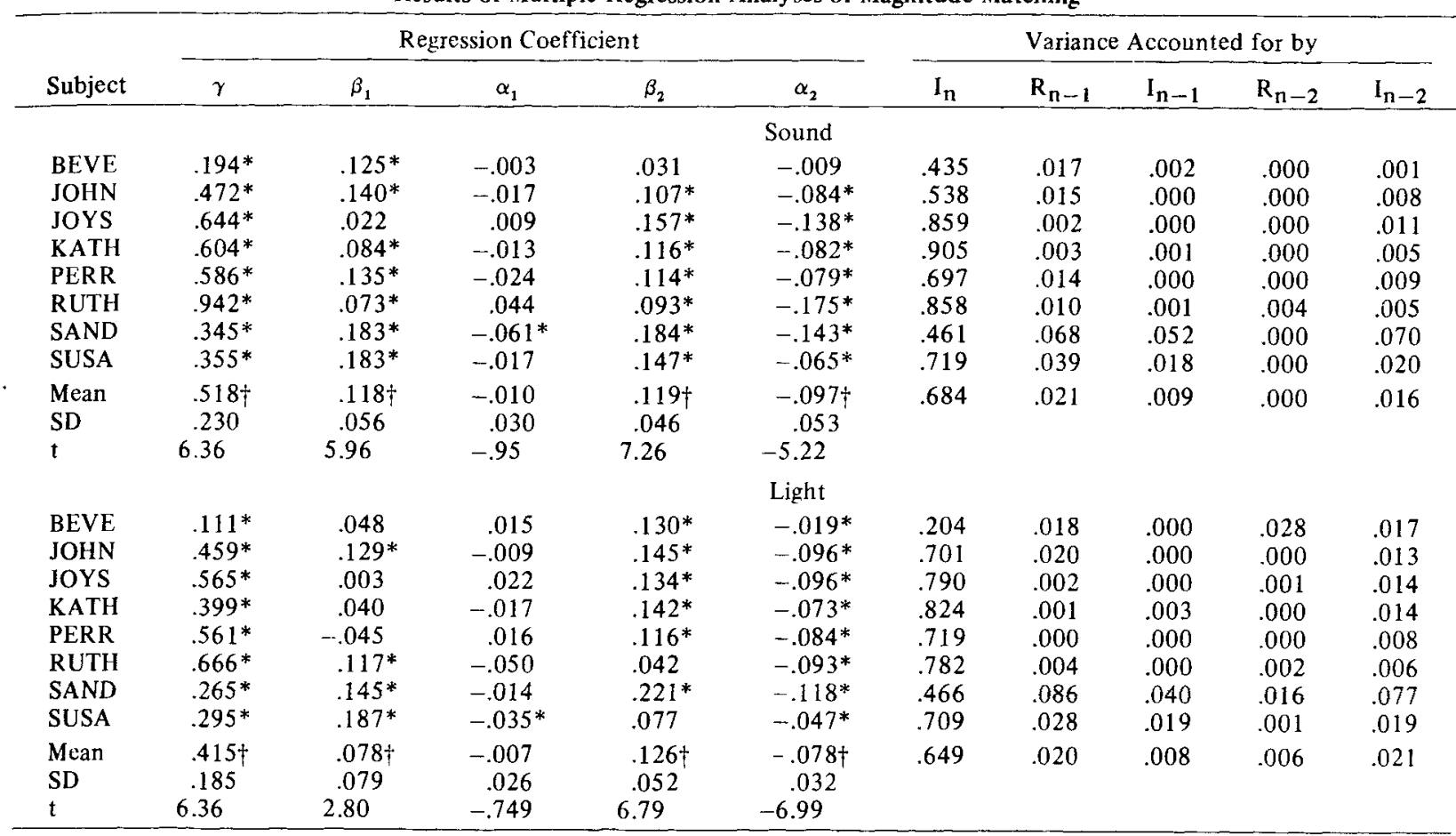

${ }^{*} p<.05$ by F test. $\quad$ tp $<.05$ by $t$ test.

less of stimulus modality, only a previous stimulus of the same modality (e.g., the stimulus two trials back in the sequence) exerts a contrast effect (negative sign of $\alpha_{2}$ ) on current responses. The fact that the effect is nearly identical for responses to lights and sounds indicates that the same-modality stimulus contrast effect is not caused by imperfect dark adaptation after exposure to the intense light stimuli between light trials. Brief sound stimuli of such intensities produce little adaptation.

The variance-accounted-for analysis is also revealing. It is clear that the current stimulus accounts for most response variance, although not nearly all of it. Of the previous stimuli and responses, clearly $R_{n-1}$ and $I_{n-2}$ account for significant amounts of $R_{n}$ variance, around $2 \%$ each. In combination with the regression coefficients, these data indicate that the most significant previous events for the current response are the immediately previous response $\left(R_{n-1}\right)$ to a stimulus of a different modality, and the stimulus two trials back $\left(I_{n-2}\right)$, of the same modality as the current stimulus. This result supports theories, such as the theory of fuzzy psychophysical judgment (Ward, 1979), that propose separate mechanisms for assimilation/response and contrast/stimulus dependencies, and tends to disconfirm theories that have both effects arising from the same mechanism.

Table 2 summarizes the results of analysis of the category matching data by estimating regression co- efficients in Equation 4. Again, $M=10$ and $N=10$ in the analyses, although results are reported only for $\mathbf{M}=2$ and $\mathbf{N}=2$. The results are identical in form to those of analyses of the magnitude matching data. The major differences are in the regression coefficients of $I_{n}$, which are considerably smaller than those for the magnitude matching data. This is consistent with the relationship usually reported between magnitude estimation and category judgment power function exponents (Marks, 1974; Ward, 1971). The other difference :- that the magnitude of the effects of previous respr ses seems to be somewhat smaller for the category matching data, especially for responses to sound stimuli, while the variance accounted for by $I_{n}$ is somewhat larger for these data, again, especially for the sound responses. It may be that allowing subjects to use any numbers they please increases response variance and the use of extraneous response system processes (see Baird \& Noma, 1975) at the expense of accuracy. The category matching data seem cleaner, and the effect of lower power function exponents is negligible since both are proportionately lower; the sound-light matching function is of the same slope in both methods.

Second-order dependencies. Jesteadt et al. (1977) showed that in magnitude estimation data the magnitude of the relation between $R_{n}$ and $R_{n-1}$ depends on the magnitude of the difference (or ratio) between $I_{n}$ and $I_{n-1}$. They found an inverted V-shaped func- 
Table 2

Results of Multiple-Regression Analyses of Category Judgments

\begin{tabular}{|c|c|c|c|c|c|c|c|c|c|c|}
\hline \multirow[b]{2}{*}{ Subject } & \multicolumn{5}{|c|}{ Regression Coefficient } & \multicolumn{5}{|c|}{ Variance Accounted for by } \\
\hline & $\gamma$ & $\beta_{1}$ & $\alpha_{1}$ & $\beta_{2}$ & $\alpha_{2}$ & $I_{n}$ & $R_{n-1}$ & $I_{n-1}$ & $R_{n-2}$ & $I_{n-2}$ \\
\hline & & & & & Sound & & & & & \\
\hline CAMI & $.289^{*}$ & .011 & .018 & $.245^{*}$ & $-.096^{*}$ & .785 & .005 & .001 & .000 & .025 \\
\hline CHOW & $.377 *$ & $.105^{*}$ & .001 & $.127^{*}$ & $-.076^{*}$ & .829 & .004 & .000 & .001 & .008 \\
\hline CLAD & $.417^{*}$ & .054 & .005 & $.118^{*}$ & $-.077 *$ & .821 & .004 & .000 & .001 & .007 \\
\hline DERK & $.269^{*}$ & .039 & -.006 & $.145^{*}$ & $-.048^{*}$ & .693 & .001 & .000 & .003 & .017 \\
\hline LILY & $.430^{*}$ & .084 & -.005 & $.107^{*}$ & $-.114^{*}$ & .692 & .001 & .000 & .006 & .020 \\
\hline PIGG & $.428^{*}$ & .046 & -.009 & $.126^{*}$ & $-.078 *$ & .813 & .001 & .001 & .000 & .010 \\
\hline TERR & $.431^{*}$ & .061 & -.002 & $.179^{*}$ & $-.112^{*}$ & .880 & .003 & .000 & .002 & .009 \\
\hline WEST & $.365^{*}$ & $.095^{*}$ & .006 & $.173^{*}$ & $-.090^{*}$ & .794 & .009 & .000 & .000 & .015 \\
\hline Mean & $.376 \dagger$ & $.062 \dagger$ & .001 & $.140 \dagger$ & $-.086 \dagger$ & .801 & .004 & .000 & .002 & .014 \\
\hline SD & .065 & .031 & .009 & .059 & .022 & & & & & \\
\hline \multirow[t]{2}{*}{$t$} & 16.39 & 5.66 & .327 & 6.68 & -11.26 & & & & & \\
\hline & & & & & Light & & & & & \\
\hline CAMI & $.281^{*}$ & $.124^{*}$ & .005 & $.143^{*}$ & $-.060^{*}$ & .748 & .013 & .000 & .000 & .017 \\
\hline CHOW & $.205^{*}$ & $.142^{*}$ & -.009 & $.099^{*}$ & $-.026^{*}$ & .684 & .034 & .000 & .000 & .006 \\
\hline CLAD & $.337^{*}$ & .069 & .027 & $.124^{*}$ & $-.055^{*}$ & .693 & .001 & .000 & .003 & .017 \\
\hline DERK & $.261^{*}$ & $.195^{*}$ & $-.043 *$ & .039 & $-.030^{*}$ & .697 & .004 & .003 & .001 & .005 \\
\hline LILY & $.269^{*}$ & $.120^{*}$ & -.002 & .065 & $-.032^{*}$ & .699 & .024 & .000 & .000 & .009 \\
\hline PIGG & $.235^{*}$ & $.088^{*}$ & -.015 & $.161^{*}$ & $-.051^{*}$ & .740 & .012 & .002 & .000 & .013 \\
\hline TERR & $.321^{*}$ & .065 & -.005 & $.098^{*}$ & $-.047^{*}$ & .817 & .003 & .000 & .000 & .005 \\
\hline WEST & $.254^{*}$ & $.161^{*}$ & -.017 & -.010 & $-.015^{*}$ & .779 & .021 & .000 & .002 & .002 \\
\hline Mean & $.270 \dagger$ & $.121 \dagger$ & -.007 & $.090 \dagger$ & $-.040 \dagger$ & .732 & .014 & .001 & .001 & .009 \\
\hline SD & .043 & .045 & .020 & .057 & .016 & & & & & \\
\hline t & 17.72 & 7.53 & -.991 & 4.50 & -7.10 & & & & & \\
\hline
\end{tabular}

${ }^{*} p<.05$ by F test. $\quad$ tp $<.05$ by $t$ test.

tion, with correlation between $\log R_{n}$ and $\log R_{n-1}$ largest when $I_{n}-I_{n-1}$ was near zero and smallest when $I_{n}-I_{n-1}$ was different from 0 . This secondorder dependency has since been found for category judgments and cross-modality matches (Ward, 1979), and has been studied for variants of these tasks (Baird, Green, \& Luce, 1980; Green, Luce, \& Duncan, 1977; Luce \& Green, 1978). The appearance of a secondorder dependency in the present data would confirm the apparent "normality" of response-response dependencies found here (see above). Figures 2 and 3 summarize the analysis for the magnitude matching and category matching data, respectively. The data points are the average of at least five (usually eight) subjects' correlation coefficients between $\log R_{n}$ and $\log R_{n-k}$ as a function of $S_{n}-S_{n-k}$ in nominal steps, $k=1,2$. For example, the top left panels of Figures 2 and 3 show average correlation coefficients of $\log R_{n}$ with $\log R_{n-1}$, where $R_{n}$ was to a sound and $\mathbf{R}_{\mathrm{n}-1}$ was to a light (thus the effects of light on sound responses). The patterns in all eight panels of Figures 2 and 3 are identical and the same as previous results for magnitude estimation, category judgment, and cross-modality matching. Both first- and secondorder response-response sequential dependencies are independent of which modality is being responded to.

Variability of response ratios. Green and Luce (e.g., 1974) have also investigated ratios of successive magnitude estimation responses as a function of $I_{n}$ and $I_{n}-I_{n-1}$. This analysis has been extended to cross-modality matches (Baird, Green, \& Luce, 1980). For magnitude estimations, the coefficient of variation (standard deviation/mean, or $\mathrm{s} / \mathrm{m}$ ) of the response ratios declines with increasing stimulus intensity and is smaller when $I_{n}-I_{n-1}$ is near zero than when it is not near zero, producing a $V$-shaped plot of $s / m$ vs. $I_{n}-I_{n-1}$ (Green et al., 1977). The data for cross-modality matches do not show this pattern clearly (Baird et al., 1980), although it is not clear why.

Figures 4 and 5 display plots of $\mathrm{s} / \mathrm{m}$ as a function of $S_{n}-S_{n-k}(k=1,2)$ for the magnitude and category matching data, respectively. As in previous analyses, responses to light and sound are analyzed separately, and with respect to both the immediately previous stimulus and response (different modality) and the stimulus and response two trials back (same modality). The magnitude-matching data show functions that resemble those of cross-modality matching data: very ragged and with minimum $\mathrm{s} / \mathrm{m}$ when $S_{n}$ is much larger than $S_{n-k}$. There is little indication of the $V$ shape typical of ordinary magnitude estimations. The category matching data show a suggestion of a decline of $s / m$ at values of $S_{n}-S_{n-k}$ near zero, although it is not as clear as in magnitude estimation data.

Green and Luce (1974) proposed that the decline 


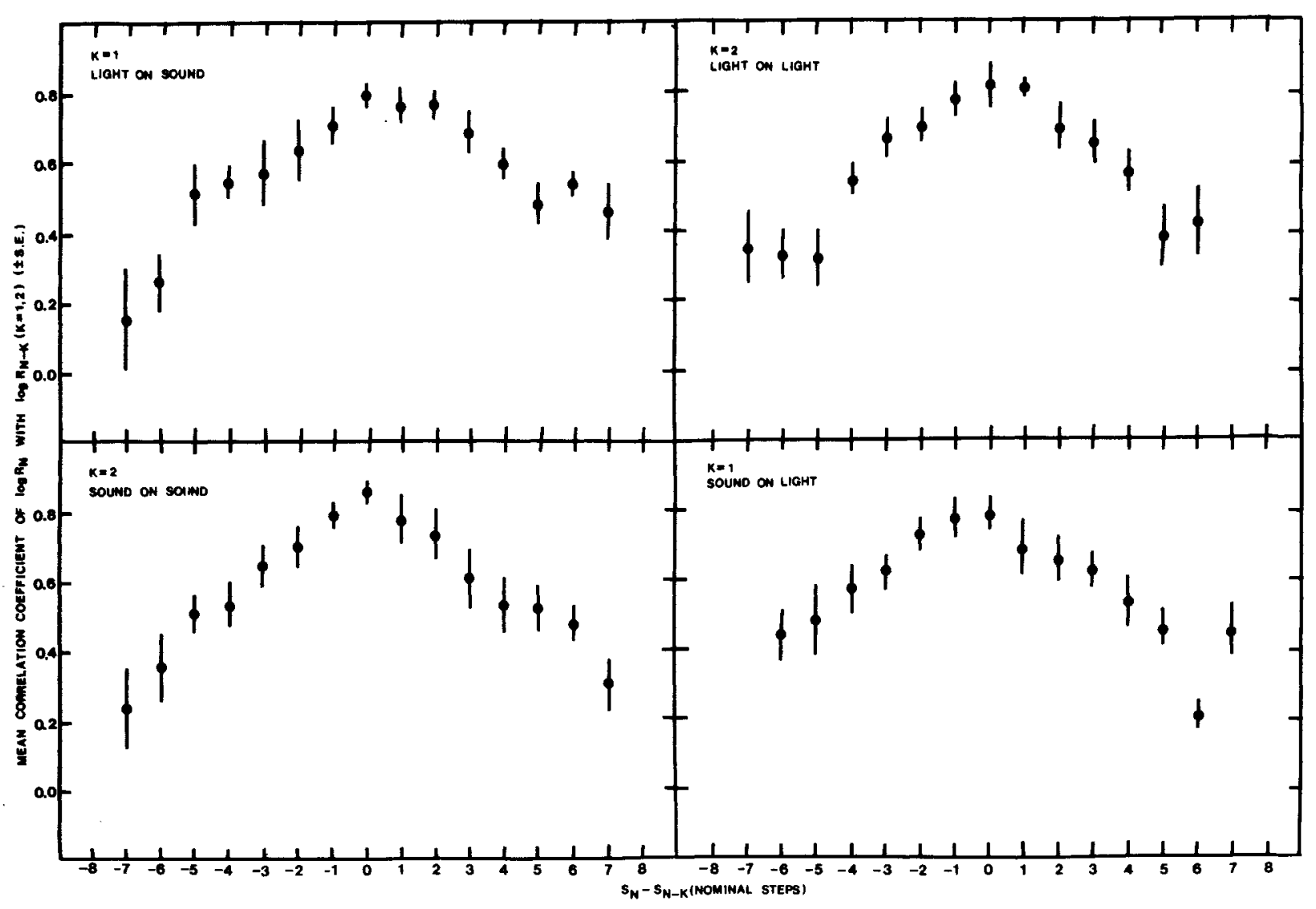

Figure 2. Results of analysis of magnitude matching data for second-order sequential dependencies.

in $\mathrm{s} / \mathrm{m}$ at values of $I_{n}-I_{n-1}$ (or $S_{n}-S_{n-1}$ ) near zero occurs because an attention band centered at $I_{n-1}$ would capture $I_{n}$ when it is near $I_{n-1}$, leading to a more precise representation of the signal (larger neural sample size) for $I_{n}$ than when $I_{n}$ is far from $I_{n-1}$ and thus outside the attention-band centered at $I_{n-1}$. A larger sample size would reduce the relative variability of $R_{n} / R_{n-1}$ for $I_{n}$ near $I_{n-1}$ relative to that for $I_{n}$ far from $I_{n-1}$. It is not clear whether this attention band is a modality-specific mechanism. If it were, we would perhaps expect to see the $V$ shape in sound-on-sound and light-on-light analyses but not in light-on-sound or sound-on-light analyses. If not, perhaps it should appear in all analyses, or, if the attention band tends to wander after a few seconds, in none of them. Since there are no clear $V$ shapes for the sound-on-light or light-on-sound curves, we can conclude that the $\mathrm{V}$-effect is probably modality specific. That there are no clear $V$ shapes for the sound-on-sound and light-on-light curves means either that the location of the attention band is unstable over the $10-15 \mathrm{sec}$ separating same-modality judgments or that the intervening judgment on a different modality somehow disrupts the tendency for the band to be located at the previous same-modality stimulus. Furthermore, the attention band cannot be the cause of both the V-shaped variability effect and the inverted- $V$ second-order sequential dependency, since the latter is normal in the present data and the former is absent. Studies of these effects in other stimulus sequences, such as S-S-L-L-S-S-L-L- ... may help clarify this situation.

\section{Conclusions}

The data reported above indicate that magnitude matching is not free from bias, although sequential dependencies should not bias the matching functions the way they do ordinary power-function exponents. The derivative method of category matching produces matching functions that are very similar to the magnitude matching functions, but are subject to the same biases. The biases themselves, the patterns of first- and second-order sequential dependencies and dependency of $\mathrm{s} / \mathrm{m}$ on the sequence of stimuli, lend support to the notion that the mechanisms responsible for assimilation of current to previous responses are different from and independent of that (those) causing contrast of current responses with previous stimuli. This, in turn, tends to disconfirm theories that derive both effects from a single mechanism. 


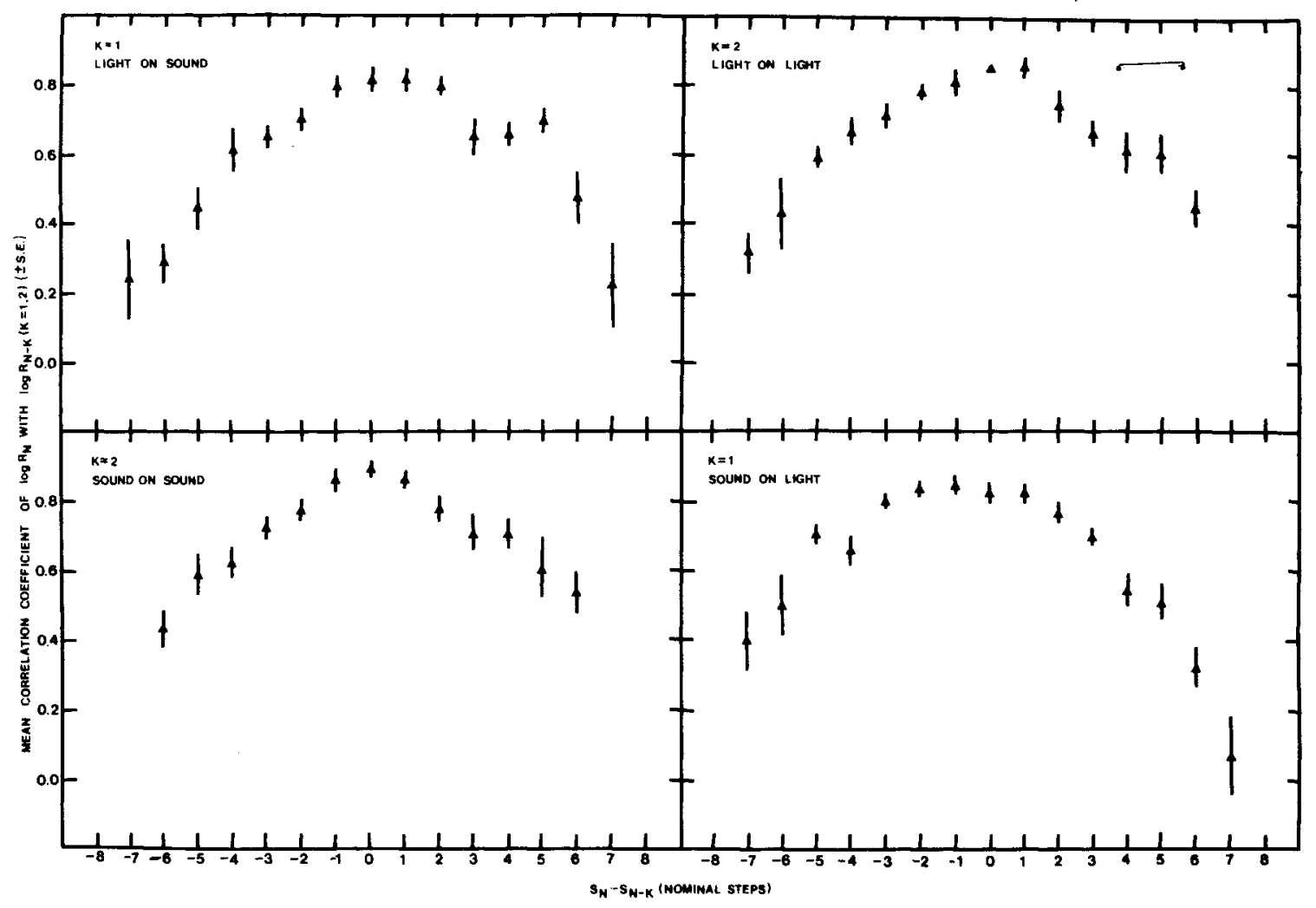

Figure 3. Results of analysis of category matching data for second-order sequential dependencies.

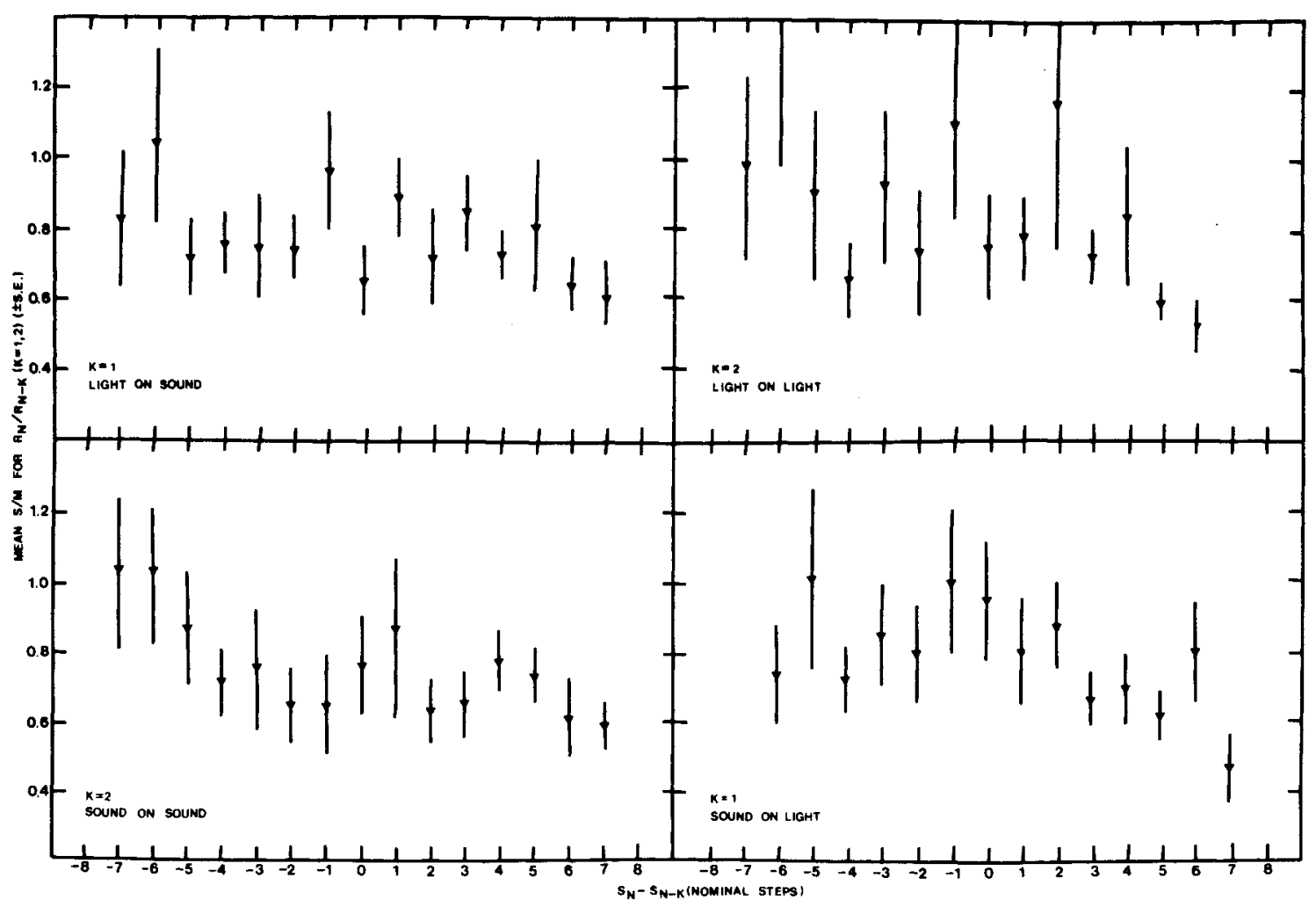

Figure 4. Results of analysis of magnitude matching data for the effects of $S_{n}-S_{n-k}$ distance on s/m for response ratios. 


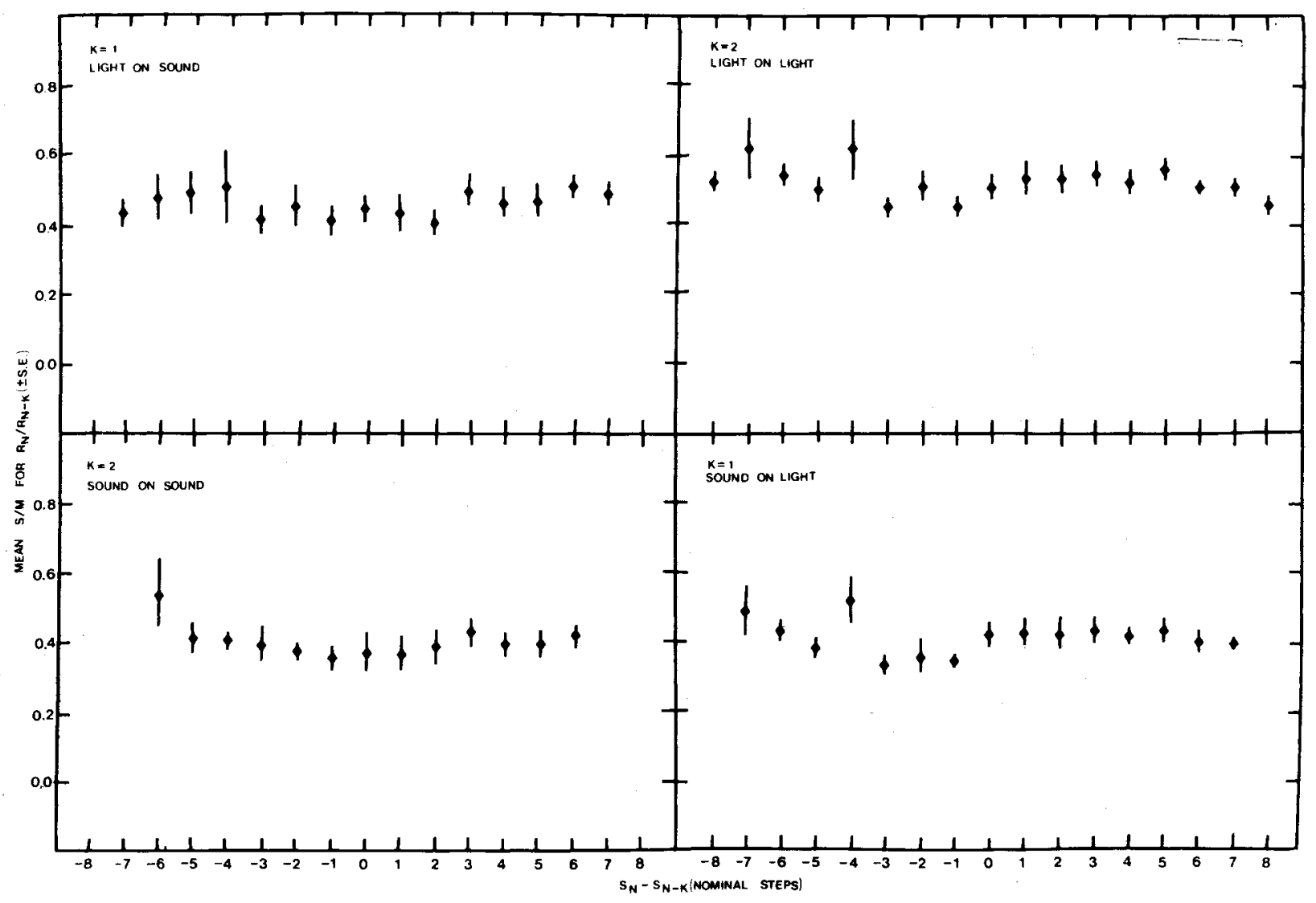

Figure 5. Results of analysis of category matching data for the effects of $S_{n}-S_{n-k}$ distance on s/m for response ratios.

Furthermore, if an attention band is responsible for the typical V-shaped dips in curves of $\mathrm{s} / \mathrm{m}$ vs. $I_{n}-I_{n-k}$, then it cannot easily also account for the observed second-order response dependencies, since the latter are present in the present data but the former are not.

\section{REFERENCES}

Baird, J. C., Green, D. M., \& LuCe, R. D. Variability and sequential effects in cross-modality matching of area and loudness. Journal of Experimental Psychology: Human Perception and Performance, 1980, 6, 277-289.

Baird, J. C., \& Noma, E. Psychological study of numbers: I. Generation of numerical responses. Psychological Research, 1975, 37, 281-297.

Green, D. M., \& Luce, R. D. Variability of magnitude estimates: A timing theory analysis. Perception \& Psychophysics, 1974, 15, 291-300.

Green, D. M., Luce, R. D., \& Duncan, J. E. Variability and sequential effects in magnitude production and estimation of auditory intensity. Perception \& Psychophysics, 1977, 22, 450456.

Jeste adt, W., LuCe, R. D., \& Green, D. M. Sequential effects in judgments of loudness. Journal of Experimental Psychology: Human Perception and Performance, 1977, 3, 92-104.

Levine, D. S., \& Grossberg, S. Visual illusions in neural networks: Line neutralization, tilt aftereffect, and angle expansions. Journal of Theoretical Biology, 1976, 61, 447-504.

Luce, R. D., Baird, J. C., Green, D. M., \& Smith, A. F. Two classes of models for magnitude estimation. Journal of Mathematical Psychology, 1980, 22, 121-148.

LUCE, R. D., \& G REEN, D. M. The response ratio hypothesis for magnitude estimation. Journal of Mathematical Psychology, 1974, 11, 1-14.

Luce, R. D., \& Green, D. M. Two tests of a neural attention hypothesis for auditory psychophysics. Perception \& Psychophysics, 1978, 23, 363-371.

Marks, L. E. On scales of sensation: Prolegomena to any future psychophysics that will be able to come forth as science. Perception \& Psychophysics, 1974, 16, 358-376.

Stevens, J. C., \& Marks, L. E. Cross-modality matching functions generated by magnitude estimation. Perception \& Psychophysics, 1980, 27, 379-389.

Stevens, S. S. Psychophysics: Introduction to its perceptual, neural, and social prospects. New York: Wiley, 1975.

WARD, L. M. Some psychophysical properties of category judgments and magnitude estimations (Doctoral dissertation, Duke University, 1971). Dissertation Abstracts International, 1971, 32, 1887B. (University Microfilms No. 71-24, 212)

WARD, L. M. Category judgments of loudness in the absence of an experimenter-induced identification function: Sequential effects and power function fit. Journal of Experimental Psychology, 1972, 94, 179-187.

WARD, L. M. Repeated magnitude estimations with a variable standard: Sequential effects and other properties. Perception \& Psychophysics, 1973, 13, 193-200.

WARD, L. M. Sequential dependencies and response range in cross-modality matches of duration to loudness. Perception \& Psychophysics, 1975, 18, 217-223.

WARD, L. M. Stimulus information and sequential dependencies 
in magnitude estimation and cross-modality matching. Journal of Experimental Psychology: Human Perception and Performance, 1979, 5, 444-459.

ZaDEH, L. A. Fuzzy sets. Information and Control, 1965, 8, 338-353.

\section{NOTES}

1. The slopes reported here are the geometric mean of the slope of the matching function (dB-dB plot) obtained from linear regression of sound on light and the reciprocal of the slope obtained from regression of light on sound. For the average functions, the average numbers were very nearly the same (1.59 and 1.60 for magnitude matching and 1.45 and 1.49 for category matching). For individual subjects, the numbers varied somewhat more (more variability in the functions) but were usually still quite close. This was the procedure followed by J. C. Stevens and Marks (1980, Appendix step 5).

2 . It is not clear what to make of the "statistical significance" of the individual regression coefficients. Strictly speaking, some conditions for the usual interpretations of the F test (e.g., independence) are not met in individual data, and others are uncertain (e.g., homoskedasticity). The approach taken here is to use both regression coefficients and variance accounted for as indexes of which effects are significant, and to rely more on tests of group averages, which can be interpreted more strictly, than on individual values. However, although there is some variation across individuals in the present data, most individual patterns are highly similar to the group averages.

\section{APPENDIX}

The predicted cross-modality matching function in terms of decibels can be derived in the following way. Consider two stimulus continua, 1 and 2 , that yield psychophysical power functions

$$
R_{1}=K_{1} I_{1}{ }^{n_{1}}
$$

and

$$
\mathbf{R}_{\mathbf{2}}=\mathbf{K}_{\mathbf{2}} \mathrm{I}_{\mathbf{2}}^{\mathrm{n}_{\mathbf{2}}} \text {. }
$$

Matching procedures $\left(R_{1}=R_{2}\right)$ result in

$$
I_{1}=K_{3} I_{2}^{n_{2} / n_{1}}
$$

Expressing Equation 3 in logarithmic form, we have

$$
\log I_{1}=\left(n_{2} / n_{1}\right) \log I_{2}+\log K_{3} .
$$

Expressions for decibels for continua 1 and 2 are

$$
N \mathrm{NB}_{1}=\mathrm{C}_{1} \log \left(\mathrm{I}_{1} / \mathrm{I}_{1}\right)
$$

and

$$
\mathrm{NdB}_{2}=\mathrm{C}_{2} \log \left(\mathrm{I}_{2} / \mathrm{I}_{2}\right)^{\prime} \text {, }
$$

where $I_{1}^{\prime}$ and $I_{2}^{\prime}$ are the respective reference levels on the two continua. Solving Equations 5 and 6 for $\log I_{1}$ and $\log I_{2}$, respectively, substituting these expressions into Equation 4, and simplifying yields

$$
\mathrm{NdB}_{1}=\mathrm{m} \mathrm{NdB}+\log \mathrm{K}_{4},
$$

where

$$
m=\left(C_{1} / C_{2}\right)\left(n_{2} / n_{1}\right)
$$

Notice that Equation 7 is that of a straight line, and, since $\mathrm{NdB}$ are logarithmic units, that of a power function with exponent $\mathrm{m}$ as well. Also, note that $\mathrm{m}$ is determined both by the ratio of the exponents of the individual power functions $\left(n_{2} / n_{1}\right)$ and by the ratio of the constants appearing in the expressions for $\operatorname{NdB}\left(C_{1} / C_{2}\right)$.

To obtain predicted matching functions for the present experiments, or for any experiments, it is necessary to substitute values of $C_{1}, C_{2}, n_{1}, n_{2}$, into Equation 8 and then insert the calculated value of $m$ into Equation 7 . When this is done using typical power function exponents for sound pressure (as continuum 1) and luminance of light (continuum 2), $\mathrm{n}_{1}=.67, \mathrm{n}_{2}=.33, \mathrm{C}_{1}=20$, and $\mathrm{C}_{2}=10$. This yields $m=1.0$. When the values of the average exponents of the separate power functions for light and sound obtained in Experiment 1 are used instead (.518 for sound and .415 for light-average $\gamma$ in Table 1$), m=1.60$. When those obtained from Experiment 2 are used (.376 for sound and .270 for light-average $\gamma$ in Table 2), $\mathrm{m}=1.42$. These are close to the values actually obtained when the linear regression in decibel coordinates is performed on the matching functions generated from the same data. 